\title{
Evidence of nuclear clustering throughout a major shell
}

\author{
B. Buck, ${ }^{*}$ A. C. Merchant, ${ }^{*}$ and S. M. Perez ${ }^{\dagger}$ \\ *Department of Physics, University of Oxford, Theoretical Physics, 1 Keble Road, Oxford OXI 3NP, United Kingdom \\ ${ }^{\dagger}$ Department of Physics, University of Cape Town, Private Bag, Rondebosch 7700, South Africa
}

(Received 10 July 2004; published 25 January 2005)

\begin{abstract}
We apply a cluster model to an extensive set of heavy even-even nuclei in the rare-earth region and determine the optimal core-cluster decomposition of each nucleus from a least-squares fit to the energy spectrum of its ground-state band. Good fits are obtained using a mass-symmetric form of the core-cluster interaction. We find that the core-cluster charge products deduced from the fits and the corresponding $B(E 2)$ values are strongly correlated, in support of an underlying cluster picture of these nuclei.
\end{abstract}

DOI: 10.1103/PhysRevC.71.014311 PACS number(s): 21.60.Gx, 23.20.Lv, 27.70.+q, 27.80. +w

\section{INTRODUCTION}

The existence of clustering (and in particular $\alpha$ clustering) in light nuclei is well established [1]. More recently a cluster model employing near-magic cores and a range of heavier clusters has given a good account of the spectra, electromagnetic properties, and exotic decay half-lives of a number of heavy even-even nuclei in the rare-earth and actinide regions [2,3]. In particular, the value of $B\left(E 2 ; 2^{+} \rightarrow 0^{+}\right)$ correlates strongly with the cluster charge, and its steep increase with the square of this charge is a highly significant indicator of the applicability of a cluster picture.

It is of considerable interest to ask whether cluster models may be applied more widely to mid-shell nuclei. There is no obvious answer to the question of what to choose for core and cluster in such nuclei. Nevertheless, a systematic application of the procedures used near closed shells to analyze the ground-state band energies can easily be extended to mid-shell regions. By requiring a best fit (in the least-squares sense) of the energies of the low-lying states, this method can reliably indicate appropriate core-cluster combinations. In particular, the clusters selected in this way lead to a good description of the highly sensitive $B\left(E 2 ; 2^{+} \rightarrow 0^{+}\right)$values in the corresponding mid-shell nuclei. Here we summarize the results of an extensive analysis of clustering in even-even nuclei in the mass range $140 \leqslant A_{T} \leqslant 200$.

\section{CLUSTER MODEL}

For the potential $V(r, R)$ at core-cluster separation $r$ we use

$$
V(r, R)=V_{N}(r, R)+V_{C}(r, R)+\frac{\hbar^{2}}{2 \mu r^{2}}(L+1 / 2)^{2}
$$

containing nuclear, Coulomb, and Langer-modified centrifugal terms. The nuclear potential, $V_{N}(r, R)$, is given the masssymmetric form [4]

$$
V_{N}(r, R)=-\left(\frac{A_{1} A_{2}}{A_{T}}\right) V_{0} h(r, x, a, R),
$$

where $A_{1}, A_{2}$, and $A_{T}$ are the core, cluster, and total masses, respectively, and the function $h$ is defined by

$$
h(r, x, a, R)=f(r, x, a, R) / f(0, x, a, R)
$$

with $f$ taking the combination of Saxon-Woods and cubed Saxon-Woods forms

$$
\begin{aligned}
f(r, x, a, R)= & {\left[\frac{x}{\{1+\exp [(r-R) / a]\}}\right.} \\
& \left.+\frac{1-x}{\{1+\exp [(r-R) / 3 a]\}^{3}}\right] .
\end{aligned}
$$

In Eqs. (2)-(4) the parameter values are kept fixed throughout at their original default values [4]

$$
V_{0}=54.0 \mathrm{MeV}, \quad a=0.73 \mathrm{fm}, \quad \text { and } \quad x=0.33 \text {. }
$$

The Coulomb potential $V_{C}(r, R)$ in Eq. (1) is taken to be that arising from a point cluster of charge $Z_{2}$ and a uniformly charged spherical core of charge $Z_{1}$ having the same radius $R$ as the nuclear potential. The total charge is $Z_{T}=Z_{1}+Z_{2}$.

\section{CHOICE OF CLUSTERIZATION}

Over the past ten years we have used three different methods to determine the optimum choice of clusterization.

We have considered maximal deviations of the sum of cluster and core binding energies from the smooth, liquid drop background-so-called D plots. In the rare-earth mass region of interest they prove ambiguous and inadequate. There is always a peak for $\alpha$ clusters, and then a "shoulder" with varying amounts of substructure that do not allow a clear identification of the light cluster charge. We choose a value in the critical range $2 \leqslant Z_{2} \leqslant 10$ suggested by cluster model analyses of observed spectra and $\mathrm{B}(\mathrm{E} 2)$ values in this region.

The cluster charge can be strongly correlated with $B(E 2$ : $2^{+} \rightarrow 0^{+}$) for the transition between the ground and first excited states. This is a very good indicator of cluster charge because of its near quadratic dependence on $Z_{2}$ and the correlation can be exploited to select the light cluster. The drawback with this method is that it does not simultaneously generate a potential radius parameter necessary to calculate a spectrum in good agreement with experiment. This must be added on by an additional fitting (which then usually leads to a good description of the low-lying spectrum).

Here (and previously in [5]) we prefer a method fitting the excitation energies of the low-lying states of the nucleus and also involving the potential radius $R$ (as explained in the 
following). This produces an optimal choice of $\left(Z_{2}, A_{2}\right)$ as well as a radius appropriate to the spectrum. The value of $B\left(E 2: 2^{+} \rightarrow 0^{+}\right)$is then a prediction of the model (instead of an input) and its systematics are in good agreement with the data. This implies that starting from the $B\left(E 2: 2^{+} \rightarrow 0^{+}\right)$ value would lead to the same clusterization.

We consider first a particular core-cluster decomposition of a given nucleus, corresponding to a cluster charge and mass $\left(Z_{2}, A_{2}\right)$. We set the global quantum number $G$ at $G=(2 n+$ $L)=4 A_{2}$ [see discussion after Eq. (10)], with $n$ the number of nodes in the radial wave function of the core-cluster relative motion, and $L$ the corresponding orbital angular momentum. Applying the cluster model then generates a band of states with $L^{\pi}=0^{+}, 2^{+}, \ldots, G^{+}$with energies determined by the core-cluster interaction. The latter is completely specified by Eqs. (1)-(5) except for the value of the radius parameter $R$.

In previous cluster model calculations of proton [6], $\alpha$ [7], and exotic [8] decay we have found that it is critically important for the potential, Eq. (1), to have its ground state, with $L=$ 0 and given $n$, at the exact experimental energy. This is a reflection of the extreme sensitivity of the tunneling integral to the position of the state with respect to the Coulomb barrier. Furthermore, we have found that the best way to achieve this energy match is by choosing the potential radius appropriately, while all other potential parameters are fixed. This is because the Bohr-Sommerfeld integral of Eq. (6) (see later) is more sensitive to $R$ than to any other single parameter. Over the entire mass range of the periodic table this approach leads to a rough dependence of $R$ on $A^{1 / 3}$. However, within a given shell, $R$ was found to vary much more slowly, but then to compensate this slow growth with a rapid change at the shell closure [9]. To maintain a unified approach, we adopt basically the same procedure here, and the weaker dependence of $R$ on $A$ within a shell is mirrored in the present work.

In our previous analyses the value of $R$ was fixed so as to reproduce the energy of a single level of the band (usually the $0^{+}$ground state). Here we take a more evenhanded approach and fix $R$ by requiring a least-squares fit to the entire spectrum $[5,10]$. Hence we substitute the experimental excitation energies $E_{L}^{*}(\exp )$ of the band into the Bohr-Sommerfeld quantization formula

$$
\begin{aligned}
& \int_{r_{1}}^{r_{2}} d r \sqrt{\frac{2 \mu}{\hbar^{2}}\left[Q_{0}+E_{L}^{*}(\exp )-V(r, R)\right]} \\
& =(G-L+1) \frac{\pi}{2}=I_{G L},
\end{aligned}
$$

where $Q_{0}$ is the $Q$ value for the breakup of the parent nucleus ground state into core and cluster, and $r_{1}$ and $r_{2}$ are the innermost classical turning points. The quantity $I_{G L}$, defined in Eq. (6), is strongly dependent on the choice of clusterization since almost all the terms present in that expression are dependent on the cluster mass $A_{2}$, or equivalently the cluster charge $Z_{2}$. The potential $V(r, R)$ contains nuclear [see Eq. (2)] and Coulomb terms, both of which are directly proportional to $A_{2}$ (when the no-dipole constraint is taken into account; see the following). The value adopted for $Q_{0}$ depends on the clusterization and hence on the mass defects of the cluster and core. The reduced mass $\mu$ is given by $A_{1} A_{2} /\left(A_{1}+A_{2}\right)$. The turning points $r_{1}$ and $r_{2}$ also depend on the clusterization and potential, and thus on $A_{2}$.

For a trial value of $R$, the left-hand side of Eq. (6) can be evaluated for each $L$, resulting in the quantities $J_{L}$. The optimal value of $R$ then minimizes the sum of squares

$$
S=\sum_{L}\left[J_{L}-I_{G L}\right]^{2}
$$

Using this optimal value of $R$, the theoretical excitation energies $E_{L}^{*}$ (theor) can be found by a further application of the Bohr-Sommerfeld relation

$$
\int_{r_{1}}^{r_{2}} d r \sqrt{\frac{2 \mu}{\hbar^{2}}\left[Q_{0}+E_{L}^{*}(\text { theor })-V(r, R)\right]}=I_{G L} .
$$

The good one-parameter fits obtained to the energies of the $J^{\pi}=0^{+}, 2^{+}, \ldots 10^{+}$states (together with the correlations with $B(E 2)$ values discussed later) strongly support the conclusion that there is evidence of clustering throughout the major shell under examination. It is likely that a number of minor physical effects have been subsumed in the optimal value of $R$, for example possible core and cluster deformations. We note, however, that any deformation of the parent nucleus itself can be explicitly calculated within the model [11]. We have found that this technique is useful in determining not only the optimal value of $R$ for a particular core-cluster decomposition but also the optimal core-cluster decomposition itself $[5,10]$. For each nucleus we thus select a range of even cluster masses $A_{2}=A_{2}(\min ), A_{2}(\min )+$ $2, \ldots, A_{2}(\max )$ and determine which one results in the deepest minimum of the fit parameter $S$ of Eq. (7).

\section{NO-DIPOLE CONSTRAINT}

In the light self-conjugate nuclei having $N=Z$ it would be sufficient to consider the $Q$ values of the various possible core-cluster combinations [12]. In the heavier nuclei, which have a neutron excess, there is an additional, and in our view more decisive, consideration. Our cluster model will generate a ground-state band of $0^{+}, 2^{+}, 4^{+}, \ldots$ states of positive parity and an excited band of $1^{-}, 3^{-}, 5^{-}, \ldots$ states of negative parity. Dipole transitions between such bands have strengths proportional to the squares of the matrix elements of the electric dipole operator, which depends on $\left(Z_{1} / A_{1}-Z_{2} / A_{2}\right)$. The values calculated from the cluster model can only agree with the near-zero $B(E 1)$ transition strengths observed experimentally in heavy nuclei, if the aforementioned matrix elements are very small. This requirement provides a strong basis for our adoption of an approximate no-dipole rule. For example, the experimental values for $B(E 2)$ are typically 100 times the appropriate Weisskopf unit (or more) whereas the values for $B(E 1)$ are typically $10^{-3}$ of the corresponding Weisskopf unit or less for ${ }^{218,220,222} \mathrm{Rn}$ and ${ }^{222,224,226} \mathrm{Ra}$ [13] and similarly for ${ }^{224,226,228,232} \mathrm{Th}$ and ${ }^{230,232} \mathrm{U}$ [14]. For a given nucleus our cluster model can get all the interband $B(E 1)$ 's right at the cost of one parameter [15], but the obvious overall inference for a binary cluster model is that the charge/mass ratios are nearly the same for core and cluster. 
Therefore for each $A_{2}$ the effective cluster charge $\left\langle Z_{2}\right\rangle$ is chosen so as to satisfy the no-dipole relations

$$
\frac{\left\langle Z_{1}\right\rangle}{A_{1}}=\frac{\left\langle Z_{2}\right\rangle}{A_{2}}=\frac{Z_{T}}{A_{T}},
$$

which are necessary to account for the observed near-vanishing of the $B(E 1)$ values between low-lying bands of opposite parity in heavy nuclei [16]. The nonintegral effective cluster charge $\left\langle Z_{2}\right\rangle$ (together with the corresponding effective $Q$ value $\left.\left\langle Q_{0}\right\rangle\right)$ is determined by averaging over a suitably weighted mixture of two clusters of the same mass $A_{2}$ and even charges $Z_{2} \geqslant\left\langle Z_{2}\right\rangle \geqslant Z_{2}-2$ bracketing $\left\langle Z_{2}\right\rangle[16]$.

This requires probabilities

$$
\begin{aligned}
P\left(Z_{2}\right) & =1 / 2\left[Z_{2}-\left\langle Z_{2}\right\rangle\right], \\
P\left(Z_{2}-2\right) & =1 / 2\left[\left\langle Z_{2}\right\rangle-\left(Z_{2}-2\right)\right] .
\end{aligned}
$$

We do not explicitly use the $Q$ value to choose core and cluster, relying rather on the quality of fit to the spectrum. So, corecluster combinations satisfying the no-dipole constraint and giving a good fit to the $0^{+}, 2^{+}, \ldots, 10^{+}$energies are preferred over those with the largest $Q$ values. We emphasize that in heavy, neutron-rich nuclei these are not one and the same, as they would be in light nuclei having $N=Z$ where the no-dipole constraint is satisfied automatically.

The effective values replace the $Z_{2}$ and $Q_{0}$ of a single cluster theory. The remaining quantities required in our calculations can be determined from the cluster mass $A_{2}$, for example, the nuclear core-cluster interaction using Eqs. (1)-(5), and the global quantum number $G$ from

$$
G=g A_{2},
$$

where $g=4$ for clusters composed of nucleons belonging to the major shell $Z=50-82$ and $N=82-126$ corresponding to the rare-earth region of interest here [2]. For consistency we therefore reject cases like ${ }_{56}^{144} \mathrm{Ba}_{88}$ where the indicated choice of cluster requires the breaking of this shell.

\section{Calculations}

We have applied the technique described here using the $L^{\pi}=0^{+}(2) 10^{+}$states of the ground-state bands of all eveneven nuclei in the mass region $140 \leqslant A_{T} \leqslant 200$ for which the corresponding excitation energies $E_{L}^{*}$ and $B(E 2)=B(E 2$ : $2^{+} \rightarrow 0^{+}$) values are known [17]. For each nucleus we investigate an extensive range of cluster masses $A_{2}$ and potential radii $R$, covering the physical regions for these quantities, that is, $A_{2}=6(2) 24$ and $4.0 \leqslant R \leqslant 8.0 \mathrm{fm}$ (we note that for $A_{2}=2$ or 4 no sensible averaging over an even $Z_{2}$ as described here is possible). In the vast majority of cases we find a single clearly defined minimum of the fit parameter $S$ of Eq. (7). In fact, the systematics of $R$ are consistent with a $\sim 5 \%$ increase of the value of the potential depth to $V_{0}=56.7 \mathrm{MeV}$ for the case of $A_{2}=6$ only. With this small adjustment in the default values of our parameters we have the remarkable result that only a single local minimum of $S$ exists in the range of $A_{2}$ and $R$ given here. Good fits to the spectra are obtained using the optimal clusters of Table I, with typical

\begin{tabular}{|c|c|c|c|c|}
\hline $\begin{array}{l}\text { Nucleus } \\
\left(Z_{T}, A_{T}\right)\end{array}$ & $\begin{array}{l}\text { Cluster } \\
\left(Z_{2}, A_{2}\right)\end{array}$ & $\begin{array}{c}R \\
(\mathrm{fm})\end{array}$ & $\begin{array}{c}B(E 2) / A_{T}^{4 / 3} \\
\quad\left(e^{2} \mathrm{fm}^{4}\right)\end{array}$ & $\begin{array}{c}\left(Z_{1} Z_{2} / Z_{T}\right)^{2} \\
\left(e^{2}\right)\end{array}$ \\
\hline$(56,142)$ & $(2.37,6.00)$ & 5.514 & 1.84 & 5.14 \\
\hline$(60,146)$ & $(2.47,6.00)$ & 5.617 & 1.98 & 5.59 \\
\hline$(60,148)$ & $(3.24,8.00)$ & 5.629 & 3.53 & 9.41 \\
\hline$(62,148)$ & $(2.51,6.00)$ & 5.659 & 1.84 & 5.82 \\
\hline$(62,150)$ & $(3.31,8.00)$ & 5.658 & 3.39 & 9.80 \\
\hline$(64,152)$ & $(4.21,10.0)$ & 5.641 & 4.34 & 15.5 \\
\hline$(64,160)$ & $(8.80,22.0)$ & 5.733 & 12.1 & 57.6 \\
\hline$(66,154)$ & $(4.29,10.0)$ & 5.683 & 5.79 & 16.1 \\
\hline$(66,160)$ & $(8.25,20.0)$ & 5.723 & 11.7 & 52.1 \\
\hline$(66,162)$ & $(8.15,20.0)$ & 5.722 & 12.0 & 51.0 \\
\hline$(66,164)$ & $(8.85,22.0)$ & 5.763 & 12.5 & 58.8 \\
\hline$(68,156)$ & $(2.62,6.00)$ & 5.782 & 3.92 & 6.32 \\
\hline$(68,158)$ & $(4.30,10.0)$ & 5.725 & 7.07 & 16.3 \\
\hline$(68,160)$ & $(5.95,14.0)$ & 5.633 & 10.0 & 29.5 \\
\hline$(68,162)$ & $(6.72,16.0)$ & 5.712 & 11.4 & 36.6 \\
\hline$(68,164)$ & $(7.46,18.0)$ & 5.739 & 12.1 & 44.1 \\
\hline$(68,166)$ & $(8.19,20.0)$ & 5.749 & 12.8 & 51.9 \\
\hline$(68,168)$ & $(8.10,20.0)$ & 5.743 & 12.5 & 50.9 \\
\hline$(68,170)$ & $(8.00,20.0)$ & 5.731 & 12.4 & 49.8 \\
\hline$(70,158)$ & $(2.66,6.00)$ & 5.820 & 4.33 & 6.54 \\
\hline$(70,160)$ & $(3.50,8.00)$ & 5.770 & 5.71 & 11.1 \\
\hline$(70,162)$ & $(4.32,10.0)$ & 5.766 & 7.93 & 16.4 \\
\hline$(70,164)$ & $(5.98,14.0)$ & 5.659 & 9.67 & 29.9 \\
\hline$(70,166)$ & $(6.75,16.0)$ & 5.737 & 11.3 & 37.2 \\
\hline$(70,168)$ & $(7.50,18.0)$ & 5.761 & 12.4 & 44.8 \\
\hline$(70,170)$ & $(7.41,18.0)$ & 5.761 & 12.1 & 43.9 \\
\hline$(70,172)$ & $(8.14,20.0)$ & 5.758 & 12.6 & 51.7 \\
\hline$(70,174)$ & $(8.05,20.0)$ & 5.740 & 12.2 & 50.7 \\
\hline$(70,176)$ & $(7.16,18.0)$ & 5.729 & 11.0 & 41.3 \\
\hline$(72,166)$ & $(4.34,10.0)$ & 5.793 & 7.59 & 16.6 \\
\hline$(72,168)$ & $(6.00,14.0)$ & 5.680 & 9.23 & 30.3 \\
\hline$(72,170)$ & $(6.78,16.0)$ & 5.755 & 10.6 & 37.7 \\
\hline$(72,172)$ & $(6.70,16.0)$ & 5.749 & 9.16 & 36.9 \\
\hline$(72,174)$ & $(7.45,18.0)$ & 5.764 & 9.88 & 44.6 \\
\hline$(72,176)$ & $(7.36,18.0)$ & 5.755 & 10.7 & 43.7 \\
\hline$(72,178)$ & $(6.47,16.0)$ & 5.711 & 9.63 & 34.7 \\
\hline$(72,180)$ & $(6.40,16.0)$ & 5.698 & 9.15 & 34.0 \\
\hline$(74,168)$ & $(3.52,8.00)$ & 5.818 & 6.95 & 11.3 \\
\hline$(74,170)$ & $(5.22,12.0)$ & 5.768 & 7.56 & 23.6 \\
\hline$(74,172)$ & $(6.88,16.0)$ & 5.769 & 12.2 & 39.0 \\
\hline$(74,180)$ & $(5.76,14.0)$ & 5.690 & 8.25 & 28.2 \\
\hline$(74,182)$ & $(5.69,14.0)$ & 5.689 & 8.05 & 27.6 \\
\hline$(74,184)$ & $(4.83,12.0)$ & 5.732 & 7.13 & 20.4 \\
\hline$(74,186)$ & $(3.98,10.0)$ & 5.691 & 6.48 & 14.2 \\
\hline$(76,182)$ & $(5.01,12.0)$ & 5.767 & 7.39 & 21.9 \\
\hline$(76,184)$ & $(4.96,12.0)$ & 5.764 & 6.12 & 21.5 \\
\hline$(76,186)$ & $(4.09,10.0)$ & 5.745 & 5.48 & 14.9 \\
\hline$(76,188)$ & $(3.23,8.00)$ & 5.824 & 4.72 & 9.59 \\
\hline$(76,190)$ & $(3.20,8.00)$ & 5.823 & 4.21 & 9.40 \\
\hline$(76,192)$ & $(2.38,6.00)$ & 5.805 & 3.70 & 5.29 \\
\hline$(78,184)$ & $(5.94,14.0)$ & 5.711 & 7.55 & 30.1 \\
\hline$(78,186)$ & $(5.03,14.0)$ & 5.779 & 5.61 & 22.2 \\
\hline$(78,188)$ & $(2.49,6.00)$ & 5.846 & 4.83 & 5.81 \\
\hline$(78,190)$ & $(2.46,6.00)$ & 5.838 & 3.20 & 5.69 \\
\hline$(78,192)$ & $(2.44,6.00)$ & 5.837 & 3.45 & 5.58 \\
\hline
\end{tabular}
results for the Hf isotopes shown in Fig. 1. Preliminary results
TABLE I. Results of optimal cluster model fits, together with $B(E 2) / A_{T}^{4 / 3}$ values [17]. See text for discussion. 
TABLE I. (Continued.)

\begin{tabular}{lcccc}
\hline \hline $\begin{array}{c}\text { Nucleus } \\
\left(Z_{T}, A_{T}\right)\end{array}$ & $\begin{array}{c}\text { Cluster } \\
\left(Z_{2}, A_{2}\right)\end{array}$ & $\begin{array}{c}R \\
(\mathrm{fm})\end{array}$ & $\begin{array}{c}B(E 2) / A_{T}^{4 / 3} \\
\left(e^{2} \mathrm{fm}^{4}\right)\end{array}$ & $\begin{array}{c}\left(Z_{1} Z_{2} / Z_{T}\right)^{2} \\
\left(e^{2}\right)\end{array}$ \\
\hline$(78,196)$ & $(2.39,6.00)$ & 5.837 & 2.46 & 5.36 \\
$(80,186)$ & $(2.58,6.00)$ & 5.895 & 2.58 & 6.24 \\
$(80,196)$ & $(2.45,6.00)$ & 5.886 & 2.02 & 5.63 \\
$(80,198)$ & $(2.42,6.00)$ & 5.884 & 1.72 & 5.53 \\
\hline \hline
\end{tabular}

of a more extensive application including $L^{\pi}=0^{+}(2) 20^{+}$ states are consistent with those presented here, although with less well-defined minima in some cases, possibly pointing to difficulties in determining the higher lying states of the ground-state band.

Having determined optimal core-cluster decompositions we next focus on the relation between these results and the independent data on experimental $B(E 2)$ 's given in Table I [17]. The expression

$$
\begin{aligned}
B(E 2) & =B\left(E 2: 2^{+} \rightarrow 0^{+}\right) \\
& \sim \frac{1}{4 \pi}\left[\frac{Z_{1} Z_{2}}{Z_{T}} \int_{0}^{\infty} r^{2} \chi_{0}^{2} d r\right]^{2} \\
& \sim \frac{1}{4 \pi}\left[\frac{Z_{1} Z_{2}}{Z_{T}} r_{0}^{2} A_{T}^{2 / 3}\right]^{2}
\end{aligned}
$$

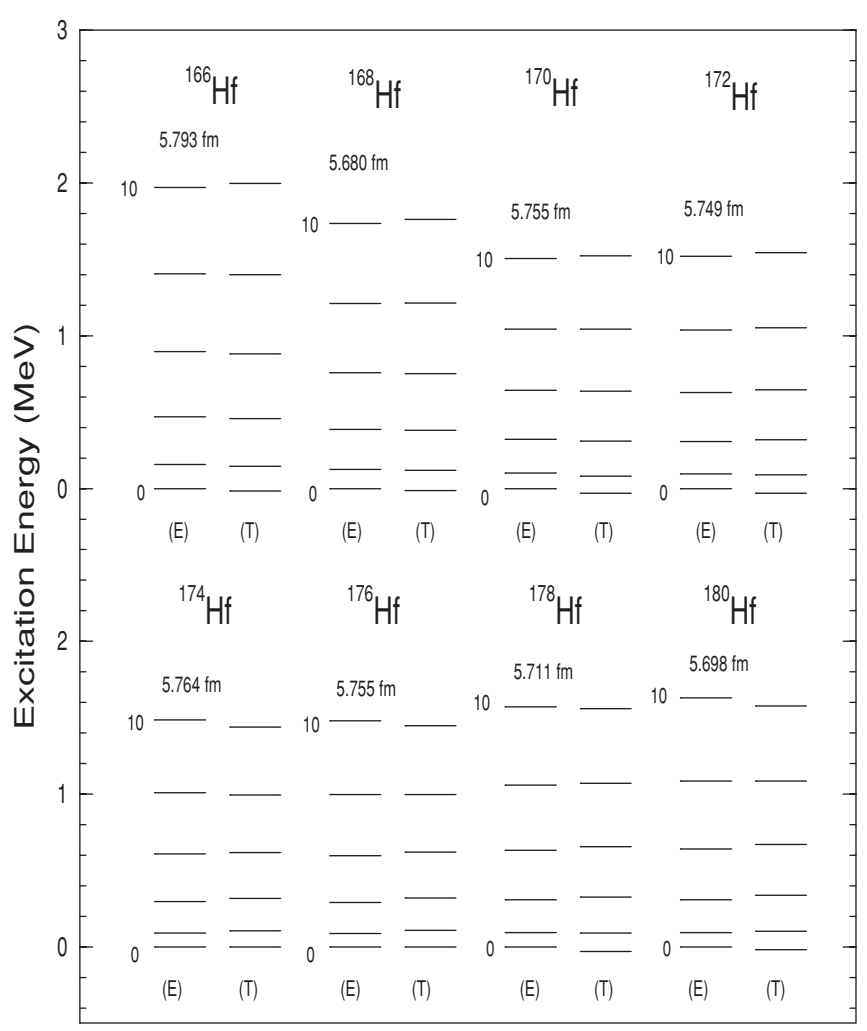

FIG. 1. Comparison of calculated (T) ground-state band excitation energies with experiment $(\mathrm{E})$ for isotopes of $\mathrm{Hf}$ from the ground state spin $0^{+}$to $10^{+}$. The core-cluster combinations used are listed in Table I. The radius parameter $R$ of Eq. (4) is given above the spectrum of each isotope.

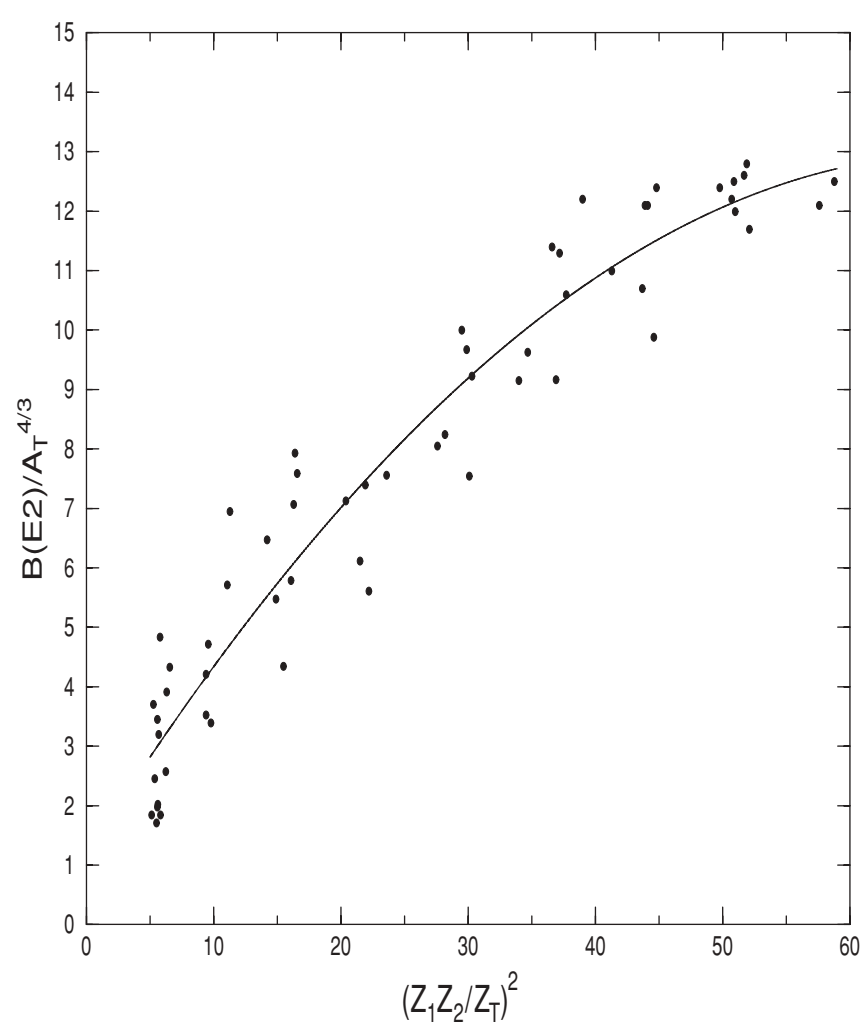

FIG. 2. Values of $B(E 2) / A_{T}^{4 / 3}$ against $\left(Z_{1} Z_{2} / Z_{T}\right)^{2}$ deduced from experimental data (full circles; see text for details). The solid line is a quadratic best fit to the data drawn to guide the eye.

is easily derived from a cluster model [18], and Fig. 2 shows that the values of $B(E 2) / A_{T}^{4 / 3}$ and the charge products $\left(Z_{1} Z_{2} / Z_{T}\right)^{2}$ calculated from the optimal core-cluster decompositions of Table I are indeed strongly correlated. Although we do not quite obtain the expected linear plot, the slopes of the chords drawn between the origin and points on the curve at $\left(Z_{1} Z_{2} / Z_{T}\right)^{2}=25.0$ and 50.0 are rather similar and result in values of $r_{0} \sim 1.42$ and $\sim 1.32 \mathrm{fm}$, respectively, thus falling in a pleasingly physical range.

The calculated $B(E 2)$ values are in good agreement with measured values and are therefore predictions arising from the model. We can also predict energies and other properties of higher $J$ states in all the nuclei we have examined here, at least up to the point where band-crossing may occur. We have successfully applied these ideas to describe and predict values for observables in isotopes of $\mathrm{Ba}$ [19], $\mathrm{Ce}$ [20], $\mathrm{Nd}$ [20], and $\mathrm{Yb}$ [5] in the rare-earth region and of $\mathrm{Po}, \mathrm{Ra}, \mathrm{Th}, \mathrm{U}$, and $\mathrm{Pu}$ [21] in the actinide region.

In conclusion we have applied a cluster model to an extensive range of nuclei spanning the mass region $140 \leqslant A_{T} \leqslant 200$. We have used a mass-symmetric core-cluster potential, with parameter values taken from an earlier cluster model analysis involving a very different database [4]. Good fits to the spectra are obtained by optimizing the core-cluster decompositions and the corresponding values of the potential radius 
parameter $R$. The charge products deduced from these fits show the expected strong correlation with experimental $B(E 2) / A_{T}^{4 / 3}$ values. The cluster model is thus found to give a consistently good description of the properties of the ground-state bands of these nuclei.

\section{ACKNOWLEDGMENTS}

S.M.P. would like to thank the UK Engineering and Science Research Council (EPSRC), the S.A. Foundation for Research, and the University of Cape Town for financial support.
[1] K. Wildermuth and Y. C. Tang, A Unified Theory of the Nucleus (Academic Press, New York, 1977), p. 1.

[2] B. Buck, A. C. Merchant, and S. M. Perez, Nucl. Phys. A652, 211 (1999); Nucl. Phys. A657, 267 (1999).

[3] B. Buck, A. C. Merchant, and S. M. Perez, Phys. Rev. Lett. 76, 380 (1996).

[4] B. Buck, A. C. Merchant, and S. M. Perez, Nucl. Phys. A614, 129 (1997).

[5] B. Buck, A. C. Merchant, V. A. McBride, and S. M. Perez, J. Phys. G 30, 1371 (2004).

[6] B. Buck, A. C. Merchant, and S. M. Perez, Phys. Rev. C 45, 1688 (1992).

[7] B. Buck, A. C. Merchant, and S. M. Perez, At. Data Nucl. Data Tables 54, 53 (1993).

[8] B. Buck, A. C. Merchant, and S. M. Perez, J. Phys. G 20, 352 (1994).

[9] B. Buck, A. C. Merchant, and S. M. Perez, Phys. Rev. C 45, 2247 (1992).

[10] V. A. McBride, M.S. thesis, University of Cape Town, 2004.

[11] B. Buck, A. C. Merchant, and S. M. Perez, Nucl. Phys. A634, 15 (1998).

[12] K. Ikeda, N. Takigawa, and H. Horiuchi, Prog. Theor. Phys. Suppl. 464 (1968).
[13] J. F. C. Cocks, P. A. Butler, K. J. Cann, P. T. Greenlees, G. D. Jones, S. Asztalos, P. Bhattacharyya, R. Broda, R. M. Clark, M. A. Deleplanque, R. M. Diamond, P. Fallon, B. Fornal, P. M. Jones, R. Julin, T. Lauritsen, I. Y. Lee, A. O. Macchiavelli, R. W. MacLeod, J. F. Smith, F. S. Stephens, and C. T. Zhang, Phys. Rev. Lett. 78, 2920 (1997).

[14] B. Ackerman, H. Baltzer, C. Ensel, K. Freitag, V. Grafen, C. Günther, P. Herzog, J. Manns, M. Marten-Tölle, U. Müller, J. Prinz, I. Romanski, R. Tölle, J. deBoer, N. Gollwitzer, and H. J. Maier, Nucl. Phys. A559, 61 (1993).

[15] B. Buck, A. C. Merchant, and S. M. Perez, Nucl. Phys. A617, 195 (1997).

[16] B. Buck, A. C. Merchant, M. J. Horner, and S. M. Perez, Phys. Rev. C 61, 024314 (2000).

[17] S. Raman, C. W. Nestor Jr., S. Kahane, and K. H. Bhatt, At. Data Nucl. Data Tables 42, 1 (1989).

[18] B. Buck, A. C. Merchant, V. A. McBride, and S. M. Perez, Phys. Rev. C 66, 067303 (2002).

[19] B. Buck, A. C. Merchant, and S. M. Perez, Nucl. Phys. A652, 211 (1999).

[20] B. Buck, A. C. Merchant, and S. M. Perez, Nucl. Phys. A657, 267 (1999).

[21] B. Buck, A. C. Merchant, and S. M. Perez, Phys. Rev. C 58, 2049 (1998). 